\author{
I. Skwira-Chalot $@$ I. Ciepał - St. Kistryn - A. Kozela • \\ W. Parol · E. Stephan
}

\title{
Invariant Coordinates in Breakup Reactions
}

\author{
Three Nucleon Force Effects
}

Received: 28 November 2016 / Accepted: 30 January 2017 / Published online: 21 February 2017

(C) The Author(s) 2017. This article is published with open access at Springerlink.com

\begin{abstract}
Systematic experimental studies of few-nucleon systems expose various dynamical ingredients which play an important role in correct description of observables, such as three-nucleon force, Coulomb force and relativistic effects. A large set of existing experimental data for ${ }^{1} H(d, p p) n$ reaction allows for systematic investigations of these dynamical effects, which vary with energy and appear with different strength in certain observables and phase space regions. Moreover, systematic comparisons with exact theoretical calculations, done in variables related to the system dynamics in a possibly direct ways is a very important tool to verify and improve the existing description of the nucleon interaction. Examples of experimental data for a breakup reaction, transformed to the variables based on Lorentz-invariants are compared with modern theoretical calculations.
\end{abstract}

\section{Introduction}

The $d p$ breakup is one of the simplest processes for testing the dynamics of three nucleons. The process is characterized by a rich kinematics of the final state which makes it selective with respect to the used model of interaction. In the wide range of energy the properties of few-nucleon systems are very well described with the use of realistic nucleon-nucleon (NN) potentials, coupled-channel (CC) calculations with realistic potential including the excitation of a single nucleon to a $\Delta$ isobar [1] or Chiral Perturbation Theory (ChPT) [2]. With increasing energy of interaction, the dynamical effects of few-nucleons, like Three Nucleon Force (3NF) [3, 4] and the relativistic component [5], start playing an important role and must be included in theoretical calculations. In case of the $d p$ breakup reaction, the Coulomb force has also a very significant influence on the cross section and also should be included in theory. The 3NF models, like Tucson Melbourn (TM99) [3] or Urbana IX (UIX) [4] are combined with realistic NN potentials to calculate the $3 \mathrm{~N}$ system observables [6]. The $3 \mathrm{NF}$ appears naturally in ChPT at a certain order while in case of CC calculations a single $\Delta$-isobar

This article belongs to the Topical Collection "The 23rd European Conference on Few-Body Problems in Physics".

I. Skwira-Chalot $(\varangle)$

Faculty of Physics, University of Warsaw, 02093 Warsaw, Poland

E-mail: izabela.skwira@fuw.edu.pl

I. Ciepał · A. Kozela · W. Parol

H. Niewodniczański Institute of Nuclear Physics PAS, 31342 Kraków, Poland

St. Kistryn

Institute of Physics, Jagiellonian University, 30348 Kraków, Poland

E. Stephan

Institute of Physics, University of Silesia, 40007 Katowice, Poland 
degree of freedom is explicitly included. Over the last few years a big effort for including all dynamical ingredients in theoretical calculations has been made. Currently calculations combining the $3 \mathrm{NF}$ and Coulomb effects are available $[7,8]$ as well as relativistic calculations including $3 \mathrm{NF}[9,10]$. High precision experimental data for the breakup process and exact theoretical calculations give an opportunity to study these very subtle dynamical effects with possible feedback on the force models. The kinematics of the ${ }^{1} H(d, p p) n$ reaction can be described in many equivalent ways, e. g. using information about the energies and emission angles of registered protons or with the Jacobi momenta, which is a very useful method for the description of reactions with three or more nucleons in the final state [11]. In order to regularly study a large database collected in a wide range of energies, a correct choice of kinematic coordinates is important. That is why an alternative way of the description of breakup reaction kinematics has been proposed [12]. The Mandelstam variables have been rewritten in a convenient way for a breakup reaction, $p+d \rightarrow p^{(1)}+p^{(2)}+n$, where all particles exist in the entrance channel - deuteron is described as a proton-neutron pair flying together with a total 4-momentum of $p_{d}$, so neglecting a relatively small bounding energy each of these particles has a 4-momentum of $p_{d} / 2$. In the exit channel there are two indistinguishable protons and one cannot say which of them, $p^{(1)}$ or $p^{(2)}$ was free (or bound) before the interaction. Based on 4-momentum of proton $p_{p}$ and deuteron $p_{d}$ in the entrance channel and 4-momentum of two protons $p_{p^{(1)}}, p_{p^{(2)}}$ and neutron $p_{n}$ in the exit channel, we define the following set of coordinates:

$-s_{p p}=\left(p_{p^{(1)}}+p_{p^{(2)}}\right)^{2}$-for protons in the exit channel,

- $s_{p n}=\left(p_{p^{(1)}}+p_{n}\right)^{2}$-for a proton-neutron pair in the exit channel (a pair with the smallest value of $\left.s_{p n}\right)$,

$-t_{n}=\left(p_{d} / 2-p_{n}\right)^{2}-4$-momentum transfer from a bound neutron in entrance channel to a free neutron in the exit channel,

$-t_{p}=\left(p_{p}-p_{p^{(2)}}\right)^{2}-4$-momentum transfer from an unbound proton to one of two protons in the exit channel (we choose the proton which wasn't used in the calculation of $s_{p n}$ ).

Instead of using rest masses of outgoing particles we propose to use variables corresponding to the kinetic energy of relative motion of two nucleons (proton-proton, proton-neutron) in the final state

$$
E_{r e l}^{p p}=\sqrt{s_{p p}}-2 m_{p}, \quad E_{r e l}^{p n}=\sqrt{s_{p n}}-m_{p}-m_{n},
$$

and the energy transfer from a bound neutron or free proton in the entrance channel to a neutron or one of two protons, respectively, in the exit channel

$$
E_{t r}^{n}=\frac{-t_{n}}{2 m_{n}}, \quad E_{t r}^{p}=\frac{-t_{p}}{2 m_{p}} .
$$

In this paper the experiment-theory comparison of $3 \mathrm{NF}$ effects is performed for cross section presented as a function of coordinates based on Lorentz-invariants.

\section{Experiment}

In order to study $3 \mathrm{NF}$ effects in a systematic way, the breakup of a deuteron in collision with a proton has been used. Experimental studies of the ${ }^{1} H(d, p p) n$ reactions with deuteron beams of energies 130 and $160 \mathrm{MeV}$ were performed at KVI in Groningen, using SALAD and BINA detectors [12-14]. The SALAD detector consisted of a three-plane MWPC and a scintilator hodoscopes: 24 horizontal detectors $(\triangle \mathrm{E})$ and 24 vertical stopping detectors (E), arranged perpendicularly to one another. It covered laboratory polar angles between $10^{\circ}$ and $40^{\circ}$ and the full range of azimuthal angles. The BINA detector consisted of two parts: Wall which is similar to SALAD and BALL which was built of almost 150 scintilators. BALL covered laboratory polar angles up to $160^{\circ}$ and the full range of azimuthal angles.

\section{Results}

Measurements of the differential cross section of the $d p$ breakup reaction at 130 and $160 \mathrm{MeV}$ have been done in a very wide range of the available phase space. The research shows that the $3 \mathrm{NF}$ plays a crucial role in the cross section. Figure 1 presents the comparison of $3 \mathrm{NF}$ effects predicted in theory (full circles) and observed in the experiment (open circles) of $d p$ breakup at $130 \mathrm{MeV}$. The $\mathrm{y}$-axis represents the ratio $\frac{\sigma_{i}-\sigma_{C D B}}{\sigma_{C D B}}$, where 

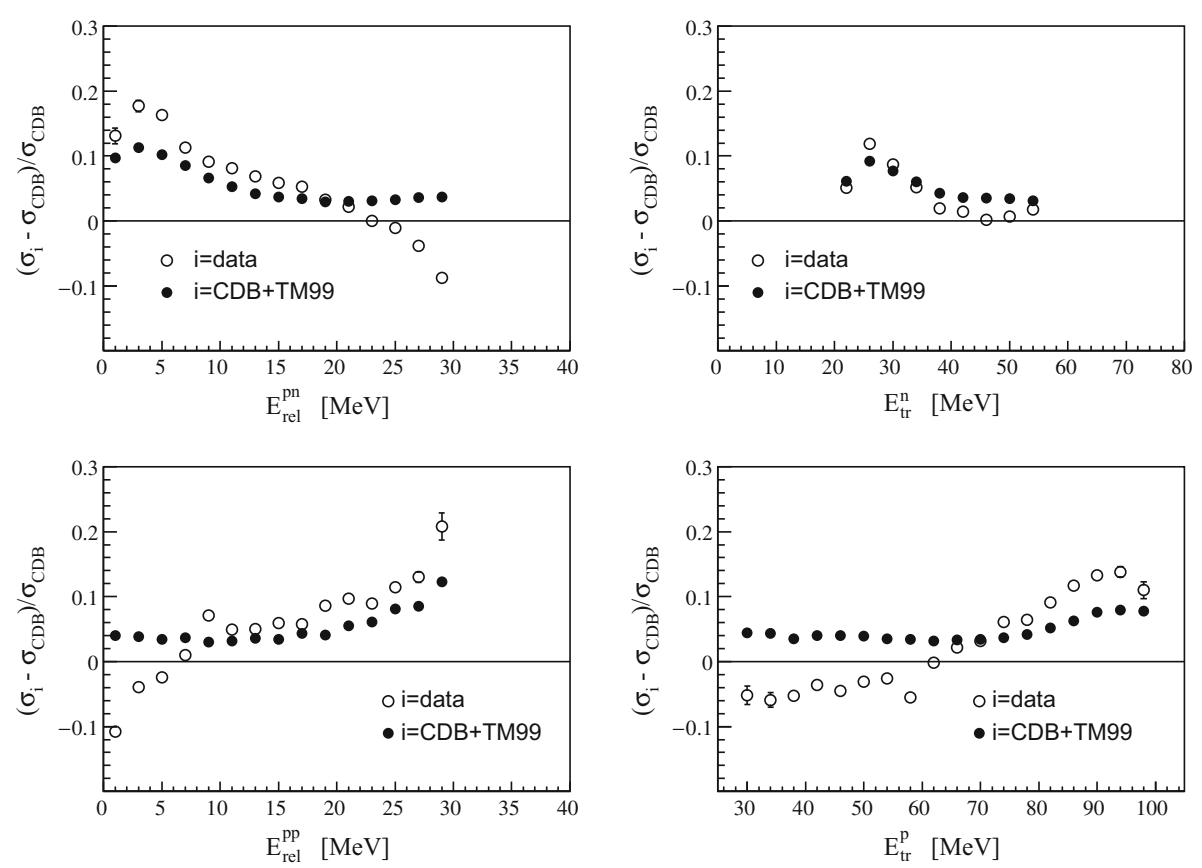

Fig. 1 Net effects of $3 \mathrm{NF}$ in the differential cross section of the $d p$ breakup at $130 \mathrm{MeV}$, presented as a function of four invariants
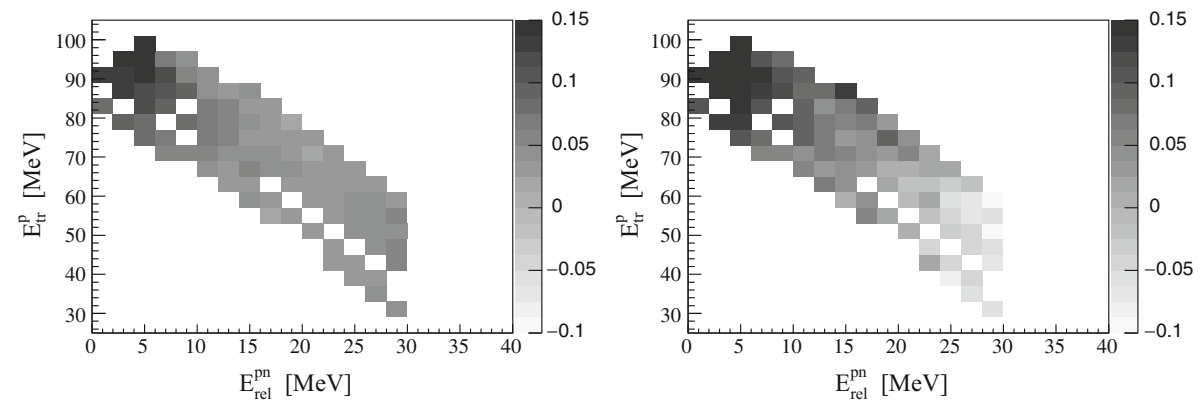

Fig. 2 Net effects of $3 \mathrm{NF}$ in the differential cross section of the $d p$ breakup at $130 \mathrm{MeV}$, presented as a function of two invariants. Left panel Difference of theoretical predictions (by Witała et al.) obtained for CD-Bonn potential with and without TM99 3NF, relatively normalized to NN calculations. Right panel Difference between experimental data and calculations with CD-Bonn alone, normalized in the same way

$\sigma_{i}$ denotes the theoretical (with the $3 \mathrm{NF}$ included) or experimental differential cross section. The theoretical calculations are based on the CD-Bonn potential with $\left(\sigma_{i}\right)$ and without $\left(\sigma_{C D B}\right)$ Tucson Melbourne 3NF (TM99) included. Generally, experimental data reveal effects beyond pure CD-Bonn NN potential, which are consistent with predicted influence of the 3NF. The disagreement between the data and theory at the largest $E_{r e l}^{p n}$, the lowest $E_{r e l}^{p p}$ and $E_{t r}^{p}$ is due to missing Coulomb force in the calculations. For better localization of $3 \mathrm{NF}$ effects two-dimensional spectra have been constructed. Figure 2 presents the net effects in function of two out of four invariants. The shapes of these spectra correspond to the acceptance of the $d p$ breakup experiment at $130 \mathrm{MeV}$ while the colors code the magnitude of the effect. Figure 3 presents theoretical predictions of the $3 \mathrm{NF}$ effects for the $d p$ breakup at $160 \mathrm{MeV}$. Calculations have been done by A. Deltuva for the Argonne V18 (AV18) potential combined with Urbana IX (UIX) 3NF [8]. The effect of 3NF is expected to be even twice so large as at energy of $130 \mathrm{MeV}$, particularly for the smallest $E_{r e l}^{p n}$ and the largest $E_{r e l}^{p p}$, which were not accessible in experiment at lower beam energy. For the $E_{t r}^{p} \geq 90 \mathrm{MeV}$ one could observe an increasing value of the $\frac{\sigma_{A V 18+U I X}-\sigma_{A V 18}}{\sigma_{A V 18}}$, which suggests that the $3 \mathrm{NF}$ in this region exists. These predictions will be soon confronted with the experimental data. 

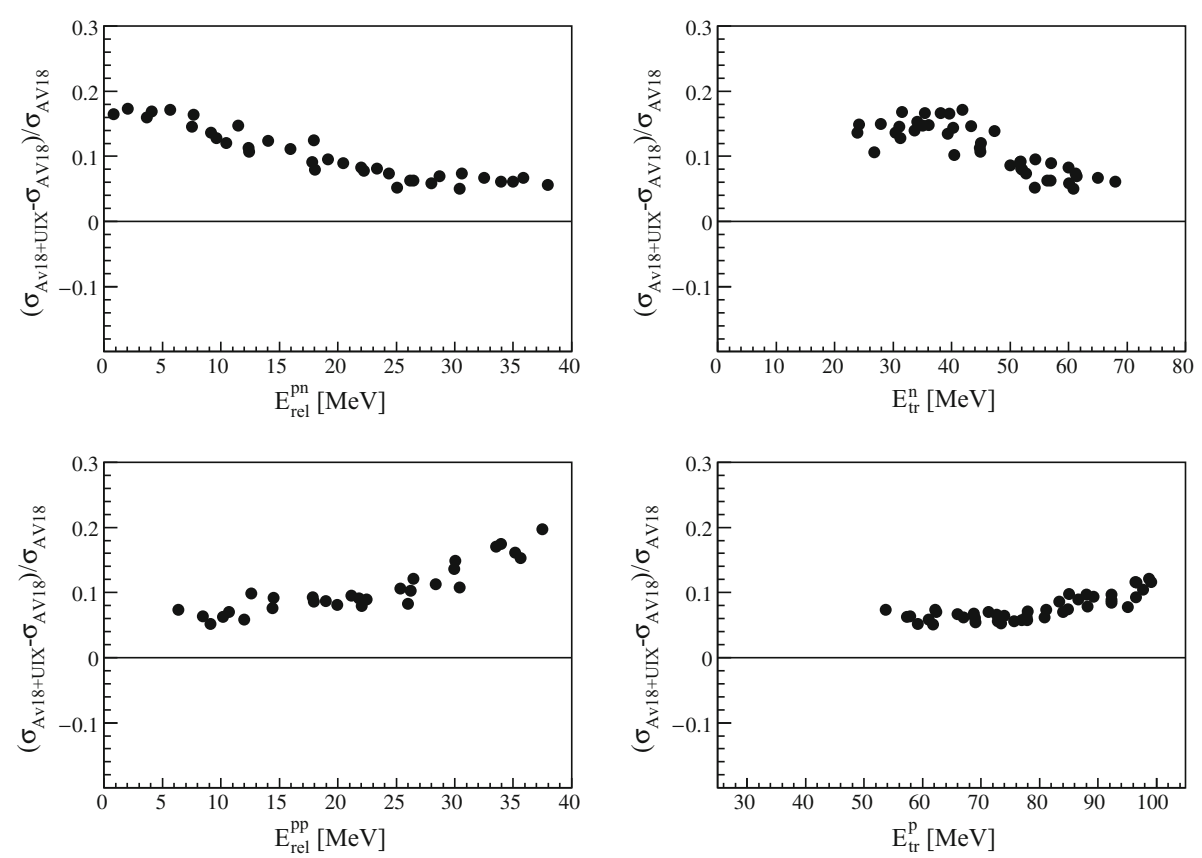

Fig. 3 Predicted net effects of $3 \mathrm{NF}$ in the differential cross section of the $d p$ breakup at $160 \mathrm{MeV}$, presented as a function of four invariants

\section{Outlook}

Systematic studies of the breakup reaction in a wide range of the phase space are very important for understanding the interaction between nucleons in few-nucleon systems. In order to verify the existing theoretical approaches, the large and exact experimental database is needed. Results presented in this paper seem to confirm the expectation that the cross section is very useful to perform systematic studies of $3 \mathrm{NF}$ effect in the regime of invariant coordinates.

Open Access This article is distributed under the terms of the Creative Commons Attribution 4.0 International License (http:// creativecommons.org/licenses/by/4.0/), which permits unrestricted use, distribution, and reproduction in any medium, provided you give appropriate credit to the original author(s) and the source, provide a link to the Creative Commons license, and indicate if changes were made.

\section{References}

1. A. Deltuva, R. Machleidt, P.U. Sauer, Realistic two-baryon potential coupling two-nucleon and nucleon- $\Delta$-isobar states: Fit and applications to three-nucleon system. Phys. Rev. C 68, 024005 (2003)

2. E. Epelbaum, Few nucleon forces and systems in chiral effective field theory. Prog. Part. Nucl. Phys. 57, 645 (2006)

3. S.A. Coon, H.K. Han, Reworking the Tucson-Melbourne three-nucleon potential. Few-Body Syst. 30, 131 (2001)

4. B.S. Pudliner, V.R. Pandharipande, J. Carlson, R.B. Wiringa, Quantum Monte Carlo calculations for A $\leq 6$ nuclei. Phys. Rev. Lett. 74, 4397 (1995)

5. R. Skibiński, H. Witała, J. Golak, Relativistic effects in exclusive neutron-deuteron breakup. Eur. Phys. J. A 30, 369 (2006)

6. H. Witała et al., Cross section minima in elastic $N d$ scattering: possible evidence for three-nucleon force effects. Phys. Rev. Lett. 81, 1183 (1998)

7. A. Deltuva, A.C. Fonseca, P.U. Sauer, New calculation schemes for proton-deuteron scattering including the Coulomb interaction. Phys. Rev. C 73, 057001 (2006)

8. A. Deltuva, Momentum-space calculation of proton-deuteron scattering including Coulomb and irreducible three-nucleon forces. Phys. Rev. C 80, 064002 (2009)

9. H. Witała et al., Relativistic effects in neutron-deuteron elastic scattering. Phys. Rev. C 71, 054001 (2005)

10. H. Witała et al., Three-nucleon force in relativistic three-nucleon Faddeev calculations. Phys. Rev. C 83, 044001 (2011)

11. W. Gloeckle, The Quantum Mechanical Few-Body Problem (Springer, Berlin, 1983)

12. Kistyn St, E. Stephan, Deutron-proton breakup at medium energies. J. Phys. G: Nucl. Part. Phys. 40, 063101 (2013) 
13. A. Ramazani-Moghaddam-Arani et al., Elastic proton-deuteron scattering at intermediate energies. Phys. Rev. C 78, 014006 (2008)

14. N. Kalantar-Nayestanaki et al., A small-angle large-acceptance detection system for hadrons. Nucl. Instr. Meth. A 444, 591 (2000) 